\title{
The Leuven Longitudinal Twin Study (LLTS): Major Findings
}

\author{
Gaston P. Beunen,' Maarten W. Peeters,' Hermine H. Maes, ${ }^{2}$ Ruth J. F. Loos, ${ }^{3}$ Albrecht L. Claessens,' \\ Catherine Derom, ${ }^{4}$ Robert Vlietinck, ${ }^{4}$ and Martine A. Thomis' \\ ' Department of Biomedical Kinesiology, Faculty of Kinesiology and Rehabilitation Sciences, Katholieke Universiteit Leuven, Belgium \\ ${ }^{2}$ Virginia Institute for Psychiatric and Behavioral Genetics, Department of Human Genetics, Virginia Commonwealth University, Richmond,Virginia, \\ United States of America \\ ${ }^{3}$ MRC Epidemiology Unit, Strangeways Research Laboratory, Cambridge, United Kingdom \\ ${ }^{4}$ Department of Human Genetics, Faculty of Medicine, Katholieke Universiteit Leuven, Belgium
}

A longitudinal study of growth and physical fitness of twins and their parents was designed in 1985. The major aims of this Leuven Longitudinal Twin Study were to quantify the genetic and environmental determination of (1) somatic characteristics, biological maturation and physical performance characteristics during the growth process, (2) the growth and developmental patterns, and (3) the covariation in somatic and performance characteristics.

Given the increased interest in the genetic determination of physical performance and the existence of the East Flanders Prospective Twin Survey, a longitudinal study of growth and physical fitness of twins and their parents was designed in 1985. The major aims of this Leuven Longitudinal Twin Study (LLTS) were: (1) to quantify the genetic and environmental determination of somatic characteristics, biological maturation and physical performance characteristics during the growth process, (2) to quantify the genetic and environmental determination of growth and developmental patterns, and (3) to quantify the genetic and environmental contributions to the covariation in somatic and performance characteristics.

Taking into account the required power of the genetic analyses to be conducted, but also the practical circumstances and financial resources, it was decided to follow 115 twin pairs from 10 to 18 years, and to study their parents once. Between 1986 and 1990 each year, between 15 and 25 twin pairs were selected from the East Flanders Prospective Twin Study. These twins were subsequently measured at semi-annual intervals for the anthropometric dimensions and at annual intervals for the performance characteristics. Only a very limited number of twin pairs (five pairs up to 15 years of age, 14 pairs at 18 years of age) discontinued the study, and another five pairs were not further included in the analyses since they did not met all inclusion criteria (for the majority excessive body fat mass).
The selection of tests and measurements was based on previous experience of the research group with longitudinal and nationwide cross-sectional growth studies. Somatic dimensions (lengths, body mass, circumferences, breadths and skinfolds), body composition (skinfolds, body mass index [BMI], waist circumference and thigh circumference), somatotype components, skeletal age, sexual maturity, and physical fitness tests (static strength, explosive strength or power, functional strength or muscular endurance of the trunk and upper-body, cardiorespiratory fitness, flexibility, running speed and speed of limb movement) were measured. Furthermore, health habits (smoking, drinking), health history, a number of sociocultural determinants and sport participation was obtained, using written questionnaires and personal interviews.

Univariate, multivariate and longitudinal models were used to analyze these data. A structural equation modeling approach was used given the superiority of this approach over other methods to estimate heritabilities. Different models were fitted to the data using the structural equation modeling package ' $\mathrm{Mx}$ '. The best fitting model was subsequently selected and maximum likelihood parameter-estimates of this model were used to determine the contribution to the phenotypic variation of the sources of variance included in the model. Such models could include additive genetic factors (A), unique or specific (E), and common environmental factors $(C)$ and/or genetic dominance (D) to explain the interindividual variation in the phenotypes. The contribution of each of these sources of variation quantifies the importance of that factor in explaining the total phenotypic variation.

The present short review summarizes the major results of the LLTS based on the manuscripts that have already been published in peer-reviewed journals. Univariate analyses of physical fitness, physical activity and strength are documented, multivariate analyses of muscularity and fat distribution are reported as well as genetic determination of the 
parameters of the growth curve. Furthermore, the heritability of somatotype components from 10 to 18 years is described, as well as the genetic and environmental determination of tracking (stability) in static and explosive strength during adolescence.

In 10-year-old twins and their parents, a simple model incorporating additive genetic effects, sometimes genetic dominance, and unique environmental factors explained the variation in physical fitness phenotypes. The genetic determination was highest for static strength, power, muscular endurance, aerobic power and flexibility ( $b^{2}$ varies between .63 and .87$)$. Balance, running speed and speed of limb movement showed somewhat lower heritabilities $\left(b^{2}\right.$ varies between .23 and .41). Common environmental factors explained a significant part of the variation in speed components and flexibility. Assortative mating was significant and positive for speed components, balance, muscular endurance of the trunk, and cardiorespiratory endurance. There is also some evidence of reduced genetic transmission for static strength, local muscular endurance of the upper-body and aerobic power. In conclusion, at this prepubertal age, genetic factors play a dominant role in explaining interindividual variation in physical performance (Maes et al., 1996).

In a subsequent cross-sectional approach it was verified if the heritabilities of static strength and power vary with age during the adolescent period. Between 10 and 18 years the additive genetic effect for static strength varied between .44 and .83 , and for explosive strength or power between .47 and .92, except at 16 years in males. Again, at each age level a simple model including additive genetic and unique environmental factors allowing for a difference in total phenotypic variance or in genetic/environmental variance in boys and girls best explained both strength characteristics during the adolescent period. Both static strength and power are thus under moderate to moderately strong genetic influence during this period of the growth process (Beunen et al., 2003).

Heritabilities for sport participation varied between .44 in females and .83 in males 15 years of age. In males a simple model including additive genetic factors and environmental factors resulted in the best fit whereas in females a common environmental factor explained also part of the phenotypic variance $\left(c^{2}=.54\right.$; Beunen \& Thomis, 1999).

Multivariate analyses of BMI, five skinfolds (biceps, triceps, suprailiac, subscapular, medial calf) and the trunk/extremity skinfold ratio revealed a general skinfold genetic factor, an extremity skinfold genetic factor, and skinfold-specific genetic factors. These results suggest that skinfolds are partly under the control of the same genetic factors, that a separate set of genes partly controls extremity skinfolds, and that other skinfold-specific genes explain a small but significant part in each of the five skinfolds. BMI is under the control of the same set of genes as skinfolds and shows high genetic correlations with trunk skinfolds. Environmental factors included a general skinfold factor and skinfold-specific factors. The bestfitting model thus included additive genetic and unique environmental factors for BMI, the five skinfolds, and the trunk/extremity skinfold ratio. All models were fairly consistent among male and female twins from 10 to 14 years of age (Beunen et al., 1998).

In another multivariate analysis, four circumferences (extended upper arm, forearm, thigh, calf) were used to unravel the genetic structure of muscularity in early adolescence (10 to 14 years). Univariate model fitting revealed that between 87 and $95 \%$ of the variance in these circumferences was explained by additive genetic factors. Multivariate analysis showed a different pattern evolving according to age and gender. In boys from 10 to 12 years of age, one general genetic factor influenced the four circumferences. With increasing age, an arm-leg model emerged, one genetic factor influencing the arm and another genetic factor influencing the leg circumferences. In young girls one genetic factor influenced the proximal (arm, and thigh circumference) and another genetic factor the distal circumferences (forearm and calf circumference). At later ages also an arm-leg model emerged. High genetic correlations indicated that genetic factors related to the four circumferences did not act independently. The age- and gender-specific changes in genetic structure suggest pubertal influences (Loos et al., 1997).

To quantify the genetic and environmental contributions to the parameters of the growth curve, individual growth curves were fitted to the semiannual measurements of stature and biologically meaningful parameters of the growth curve were derived. A model including additive genetic and unique environmental factors, allowing for a difference in total variance or in genetic/environmental variance components in males and females best explained most of the growth characteristics. For the timing and growth velocity of the adolescent growth spurt, no gender heterogeneity was observed, and the genetic (.89 to .93) and specific environmental (.07 to .11) contributions were equal in both sexes. For stature at take-off, stature at peak height velocity, and adult stature, gender heterogeneity was observed and different models appeared to result in the best fit for boys and girls. For height at peak height velocity and adult height, the significant contribution of a common environmental factor in boys was noteworthy. For percentage of adult stature and distances between relevant time points of the growth curve, a nonscalar effect was observed, indicating that different genes affect these characteristics in boys and girls. Most of the growth parameters of the adolescent period are under strong genetic control. Gender heterogeneity is present for stature and for a number of derived growth characteristics (Beunen et al., 2000).

The heritability of the somatotype (ST), a three dimensional index of human physique, was studied 
from 10 to 18 years of age. A model including additive genetic, specific and common (familial) environmental sources of variance which were shared by the three components (Ac, $\mathrm{Cc}$ and $\mathrm{Ec}$ ) as well as component-specific addiditive genetic (As) and unique environmental (Es) sources of variance fitted the data best at all measurement occasions. More than half of the total variation in all three ST components was explained by the common sources of variance (Ac, Cc and $\mathrm{Ec}$ ) stressing the need to study the heritability of the ST as a multivariate construct. Before the age of 14 , no significant sex differences in genetic and environmental determination of the three components were observed. From 14 years onwards, gender differences in the contribution of the different sources of variance became significant, supporting the need to control for age differences and the need to test for gender differences. For the endomorphy component, which describes the relative degree of fatness, heritabilities around .80 to $.90 \%$ were found up to 13 years of age. After 14 years of age, which is close to the age of peak height velocity of the growth spurt in boys (14.2 years in the LLTS), heritability of the endomorphy remained close to .80 for girls, but dropped to about .20 to .30 in boys. For the mesomorphy component, which reflects muscularity and bone mass, heritabilities between .40 and .50 were observed before 14 years of age, after which they rose close to .70 in boys and remained around .40 to .50 in girls. The third component, ectomorphy, representing the relative linearity of physique, had a heritability of around .70 in girls from 10 to 18 years of age, in boys however heritability dropped to .30 to .40 in boys after 13 years of age (Peeters et al., 2003).

The observed phenotypic stability of explosive and static strength during adolescence was studied in order to partial out the genetic from the environmental mediation of this stability. In order to control for differences in maturity status, which is known to influence motor performance, the data of the measurement occasions that were based on chronological age, were realigned on the age of peak-height velocity (PHV).

For explosive strength, as measured by the vertical jump test (VTJ), additive genetic sources of variance were found to be the main cause of phenotypic stability during adolescence: the same additive genetic sources of variance that explained a significant amount of variance 1 year before age at PHV (APHV-1) still explained $36.8 \%$ and $49.9 \%$ of the variance 3 years after age at $\operatorname{PHV}(\mathrm{APHV}+3)$. Environmental sources of variance explained less than $1 \%$ of the stability over the whole age range. At APHV+3 the total amount of variance explained by additive genetic factors explaining variance at previous measurement occasions was $56.4 \%$ and $62.8 \%$ for boys and girls respectively. For E the corresponding values were $15.3 \%$ and $7.9 \%$. Thus it was concluded that additive genetic sources of variance were the most important cause of the observed phenotypic stability in explosive strength (Peeters et al., 2005a).

For static strength, as measured by arm pull (ARP), additive genetic factors explaining variation at APHV-1 years still explained around $15 \%$ of the total variance at $\mathrm{APHV}+3$ for both boys and girls. In boys and girls respectively, unique environmental factors accounted for $3.5 \%$ and less than $1 \%$ of the stability over the entire age range, thus also suggesting that virtually only additive genetic factors explain the stability over the entire age range. However the total proportion of variance explained by transmitted additive genetic variance at APHV+3 equated $44.2 \%$ in boys and $22.4 \%$ in girls. The total proportion of transmitted variance by unique environment at $\mathrm{APHV}+3$ was $31.2 \%$ and $44.5 \%$ in boys and girls, respectively. Thus both additive genetic and environmental causes of variance explain the phenotypic stability in ARP. An increase in stability is seen after APHV in both sexes. In boys this increase can be attributed to both additive genetic and unique environmental transmitted variance, while in girls the increased stability appears to be mainly caused by unique environmental transmission (Peeters et al., 2005b).

From the above, it is clear that the data collected during this longitudinal study (LLTS) adds significantly to our knowledge about the genetic and environmental determinants of somatic dimensions, body composition, physical activity and fitness and especially their growth and developmental patterns. In the era of the search for genetic markers of complex traits, influenced by multiple genes, it appears that such a longitudinal approach is of major importance to unravel the genetic architecture of these multifactorial phenotypes. In order to attain large enough sample sizes to provide evidence of specific gene actions, efforts should be made by research groups to collaboratively explore data that have been collected longitudinally in twins or members of the same family.

\section{References}

Beunen, G., Maes, H. H., Vlietinck, R., Malina, R. M., Thomis, M., Feys, E., Loos, R., \& Derom, C. (1998). Univariate and multivariate analysis of subcutaneous fatness and fat distribution in early adolescence. Behavior Genetics, 28, 279-288.

Beunen, G., \& Thomis, M. (1999). Genetic determinants of sport participation and daily physical activity. International Journal of Obesity, 23, S55-S63.

Beunen, G., Thomis, M., Maes, H. H., Loos, R., Malina, R. M., Claessens, A. L., \& Vlietinck, R. (2000). Genetic variance of adolescent growth in stature. Annals of Human Biology, 27, 173-186.

Beunen, G., Thomis, M., Peeters, M., Maes, H. H., Claessens, A. L., \& Vlietinck, R. (2003). Genetics of strength and power characteristics in children and adolescents. Pediatric Exercise Science, 15, 128-138. 
Loos, R., Thomis, M., Maes, H. H., Beunen, G., Claessens, A. L., Derom, C., Legius, E., Derom, R., \& Vlietinck, R. (1997). Gender-specific regional changes in genetic structure of muscularity in early adolescence. Journal of Applied Physiology, 82, 1802-1810.

Maes, H. H., Beunen, G. P., Vlietinck, R. F., Neale, M. C., Thomis, M., Vanden Eynde, B., Lysens, R., Simons, J., Derom, C., \& Derom, R. (1996). Inheritance of physical fitness in 10-yr-old twins and their parents. Medicine and Science in Sports and Exercise, 28, 1479-1491.

Peeters, M. W., Thomis, M. A., Claessens, A. L., Loos, R. J. F., Maes, H. H. M., Lysens, R., Vanden Eynde, B., Vlietinck, R., \& Beunen, G. (2003). Heritability of somatotype components from early adolescence into young adulthood: A multivariate analysis on a longitudinal twin study. Annals of Human Biology, 30, $402-418$.

Peeters, M. W., Thomis, M. A., Maes, H. H. M., Loos, R. J. F., Claessens, A. L., Vlietinck, R., \& Beunen, G. P. (2005a). Genetic and environmental causes of tracking in explosive strength during adolescence. Behavior Genetics, 35, 551-563.

Peeters, M. W., Thomis, M. A., Maes, H. H. M., Beunen, G. P., Loos, R. J. F., Claessens, A. L., \& Vlietinck, R. (2005b). Genetic and environmental causes of tracking in static strength during adolescence. Journal of Applied Physiology, 99, 1317-1326. 